psychopraxis.neuropraxis 2016 · 19:109-114 DOI 10.1007/s00739-016-0334-x

Online publiziert: 11 . August 2016

(c) Der/die Autor(en) 2016. Dieser Artikel ist eine Open-Access-Publikation.

CrossMark

\title{
Thomas Brücke
}

Wien, Österreich

\section{Parkinson: L-Dopa nach wie vor wirksamste Therapie}

Therapie klinisch nur schwer praktikabel war. Große Bedeutung hatte auch die Beschreibung der Wirkungsverstärkung von L-Dopa durch den Decarboxylasehemmer Benserazid durch Birkmayer u. Mentasti (1967). Durch diese Kombination wird der Abbau von L-Dopa in der Peripherie vermindert, wodurch die orale L-Dopa-Dosis gesenkt und damit auch eine Reduktion der Nebenwirkungen erreicht werden kann.

L-Dopa wird seither nur in Kombination mit einem Decarboxylasehemmer (Benserazid im Madopar ${ }^{\circledR}$; Carbidopa im Sinemet ${ }^{\circledR}$ bzw Nacom $^{\circledR}$ ) angewendet. (Wenn im Folgenden von der „LDopa-Therapie“ die Rede ist, ist damit immer die Kombination von L-Dopa + Decarboxylasehemmer gemeint!)

Seit damals gilt die L-Dopa-Therapie als die wirksamste Behandlung der Parkinson-Erkrankung und als „Goldstandard“ an dem sich alle anderen Medikamente messen müssen.

Trotz der ausgezeichneten Wirkung von L-Dopa auf die Hauptsymptome der Erkrankung, wurde allerdings bald klar, dass auch diese Therapie ihre Probleme und Grenzen hat. Die Einnahme über einen längeren Zeitraum kann vor allem bei jüngeren Patienten zum sog. L-Dopa-Langzeitsyndrom, zu Schwankungen der Beweglichkeit in Abhängigkeit von der Medikamenteneinnahme („wearing off“) bzw. - unabhängig von der Medikamenteneinnahme („on-off“) - zu nächtlichen und morgendlichen „offs“ und überschüssigen Bewegungen (Dyskinesien) führen. Es wurden deshalb im Lauf der Jahre eine Reihe anderer Medikamente in die Therapie eingeführt, die zum Teil die L-Dopa-Wirkung verstärken bzw. verlängern (MAO-B-Hemmer, COMT-Hemmer) oder die Wirkung von Dopamin an den Dopamin-Rezeptoren nachahmen (Dopaminagonisten). Mit diesen Therapien können vor allem die Wirkungsschwankungen unter der L-Dopa-Therapie bis zu einem gewissen Grad verbessert werden. Die Kombination verschiedener Medikamente ermöglicht auch eine Reduktion der L-DopaDosis und kann damit in vielen Fällen die Dyskinesien vermindern.

\section{Fallbericht 1}

2010 bemerkte die zu dem damaligen Zeitpunkt 47-jährige Patientin erstmals, dass sie das linke Bein etwas nachzog und sich die Zehen links verkrampften. Etwa gleichzeitig erkrankte ihre Mutter schwer und verstarb ein halbes Jahr später, was bei der Patientin eine depressive Verstimmung auslöste. Kurz später fiel auf, dass der linke Arm beim Gehen nicht richtig mitschwang und eine gewisse Verlangsamung der Fingerbewegungen bestand. Deshalb wurde erstmals eine neurologische Untersuchung durchgeführt, die den Verdacht auf eine Erkrankung an M. Parkinson ergab. Die Diagnose wurde durch eine DAT-SPECT- (DopaminTransporter-SPECT-)Untersuchung bestätigt, die Hinweise für eine rechtsbetonte Degeneration dopaminerger Nervenendigungen vor allem im Putamen erbrachte. Eine MRI-Untersuchung des Schädels war unauffällig.

Es wurde daraufhin der Dopaminagonist Pramipexol ret. (Sifrol $\operatorname{retard}^{\circledR}$ ) in langsam steigernder Dosis bis $1,05 \mathrm{mg} / \mathrm{Tag}$ verschrieben. Frau O. bemerkte eine leichte Besserung der Fingerbeweglichkeit und erhielt wegen Schmerzen in der linken Schulter Physiotherapie. Die Symptome im linken Bein veränderten sich nicht. keit hoher oraler Dosen durch Cotzias in den USA (1967), da die intravenöse 
Mitte 2011 berichtete Frau O. über ein zeitweise auftretendes Ruhezittern des linken Arms. In der neurologischen Untersuchung fiel eine leichte Hypomimie sowie eine leichte linksseitige rigidhypokinetische Parkinson-Symptomatik auf.

Auf nähere Befragung gab Frau O. an, dass sowohl eine Großtante mütterlicherseits, als auch der Großvater väterlicherseits an Parkinson erkrankt waren. Die Pramipexol ret. Dosis wurde auf 2,1 mg ret/Tag. erhöht und die Symptome dadurch für ein halbes Jahr zufriedenstellend gebessert.

Bis Ende 2012 war wegen neuerlicher Zunahme der Beschwerden Pramipexol ret. auf 3,15 mg/Tag erhöht worden, außerdem war der MAO-B-Hemmer Rasagilin (Azilect $^{\circledR}$ ) zusätzlich in die Therapie eingeführt worden. Da damit nicht mehr das Auslangen gefunden wurde, wurde zu diesem Zeitpunkt - etwa 2 Jahre nach Diagnosestellung erstmals L-Dopa verschrieben. Mit 3-mal $100 / 25 \mathrm{mg} /$ Tag wurde eine deutliche Verbesserung der Muskelsteifigkeit und des Zitterns erreicht.

Wegen einer schweren Gonarthrose war das Gehen aber so beeinträchtigt, dass im Mai 2014 eine Kniegelenkendoprothese implantiert werden musste. Es kam zu Komplikationen aufgrund einer lokalen Infektion des Implantats. Deshalb musste die Patientin insgesamt viermal operiert werden und konnte sich über Monate hindurch nur im Rollstuhl fortbewegen.

Da sich unter 3,15 mg Pramipexol ret/Tag eine starke Neigung zu nächtlichen Computerspielen entwickelt hatte und damit eine Schlafverkürzung auf teilweise vier Stunden verbunden war, musste die Dosis wieder reduziert werden.

Etwa zur selben Zeit kam es dann zu zunehmenden Fluktuationen mit Onoff-Phasen. In den Off-Phasen, die besonders in den Stunden nach dem Mittagessen sehr ausgeprägt waren, kam es häufig zu schmerzhaften Verkrampfungen der Zehen links. Das Aufstehen aus dem Sitzen war nur noch schwer möglich. Beim Versuch wegzugehen, blieb Frau O. am Boden „kleben“. Sie konnte nur mit kleinen Trippelschritten gehen, und es bestand eine starke Zugtendenz nach vorne. In den On-Phasen bestand praktisch keine Parkinson-Symptomatik.

Wegen einer zunehmenden Verkürzung der L-Dopa-Wirkung steigerte die Patientin selbst die Dosis auf 5-mal 200/50 mg täglich. Nach weiterer Therapieeskalation auf eine Kombination von 4-mal L-Dopa $150 \mathrm{mg} /$ Carbidopa 37,5 mg/Entacapon $200 \mathrm{mg}$ $\left(\right.$ Stalevo $150^{\circledR}$ ) plus 4-mal 100/25 mg L-Dopa/Tag wurde die Patientin zunehmend dyskinetisch. In den On-Phasen war die Stimmungslage hypoman angehoben und die Patientin fühlte sich überdreht und stark angetrieben, um kurz darauf in der Off-Phase in eine schwere Depression zu verfallen.

Die Off-Phasen waren unter dieser Therapie ausgeprägter als vorher! Es wurde die Medikation deshalb wieder auf eine L-Dopa-Therapie von 4-mal $200 \mathrm{mg} /$ Tag reduziert und abends $25 \mathrm{mg}$ des atypischen Neuroleptikums Quetiapin $\left(\right.$ Seroquel ${ }^{\circledR}$ ) in die Therapie eingeführt. Damit kam es durch einige Monate hindurch wieder zu einer deutlichen Verbesserung der Gesamtsituation. Vier Monate später nahmen die OffPhasen aber wieder deutlich $\mathrm{zu}$ und betrugen insgesamt 6 Stunden täglich. Die zusätzliche Gabe von Entacapon $\left(\right.$ Comtan $\left.^{\circledR}\right)$ führte zur Verlängerung der On-Phasen und zu „sanfteren“ Abfällen der Beweglichkeit, sodass die Patientin mit ihrem Zustand relativ zufrieden war.

Fünf Monate später traten aber heftige Durchfälle auf (eine häufige Nebenwirkung der COMT-Hemmer), die nach Pausieren von Entacapon wieder sistierten und nach neuerlicher Einnahme sofort wieder auftraten. Deshalb musste Entacapon endgültig abgesetzt werden, was wieder eine starke Verschlechterung der Parkinson-Symptomatik mit sich brachte.

Frau O. war nur noch vier Stunden tagsüber gut beweglich und auch nachts motorisch sehr schlecht. Es wurde deshalb Ende 2015 ein Therapieversuch mit dem COMT-Hemmer Tolcapon (Tasmar $^{\circledR}$ ) 3-mal $100 \mathrm{mg}$ täglich begonnen. Die Behandlung mit Tolcapon macht vierzehntägige Blutabnahmen zur Kontrolle der Leberwerte notwendig! Durch Tolcapon kam es sowohl tagsüber als auch nachts zu einer deutlichen Verbesserung der Motorik, sodass nur noch gegen Mittag eine zweistündige Off-Phase auftrat, in der Frau O. aber so beweglich war, dass sie ohne Hilfe gehen konnte.

Dieser verbesserte Zustand hält bisher an, und die regelmäßigen Kontrollen der Leberwerte sind in Ordnung. Die aktuelle medikamentöse Einstellung war zuletzt: L-Dopa 200/50: 1/2 - 1/2 - 1/2 - (1/2) täglich; L-Dopa LT: $1-1-1-0$ täglich; Rasagilin 1 mg: 1 morgens; Tolcapon 100 mg: $1-1-1$ - 0 täglich; Pramipexol ret. 1,05 mg: 1 morgens; Quetiapin 25 mg: 2 abends. Prinzipiell wäre bei der Patientin auch die Indikation zu einer tiefen Hirnstimulation gegeben.

\section{Fallbericht 2}

2006 suchte der damals 70-jährige Patient, Herr L., erstmals eine neurologische Ambulanz wegen eines seit zwei Jahren bestehenden Ruhezitterns der rechten Hand auf. Außer dem mäßigen Ruhetremor zeigte sich auch ein leichter Rigor und eine verminderte Mitbewegung des rechten Arms beim Gehen. Ansonsten war die neurologische Untersuchung unauffällig, insbesondere bestand kein Hinweis für eine Brady- bzw. Hypokinese. Herr L. war in sehr gutem Allgemeinzustand und wirkte biologisch jünger. In einer Dopamin-Transporter-Untersuchung im SPECT wurde der Verdacht einer dopaminergen Läsion im Striatum bestätigt und der Patient primär auf den MAO-B-Hemmer Rasagilin eingestellt. Da sich unter dieser Therapie der Tremor nicht verbesserte, wurde $2007 \mathrm{zu}$ sätzlich der Dopaminagonist Pramipexol ret. verschrieben und bis zu einer Dosis von 2,1 mg/Tag gesteigert. Auch diese Behandlung führte zu keiner Reduktion des Tremors.

Herr L. fühlte sich sonst aber nicht beeinträchtigt. Pramipexol ret. wurde anschließend wieder abgesetzt und eine Einstellung auf Budipin (Parkinsan ${ }^{\circledR}$ ), (eine anticholinerg und antiglutamaterg wirkende Substanz mit guter Wirkung auf den Tremor) in einer Dosis von primär 3-mal $10 \mathrm{mg} /$ Tag vorgenommen. Da Budipin zu einer Qt-Verlängerung führen kann, und die Verschreibbarkeit 
deshalb nur unter regelmäßiger EKGKontrolle möglich ist, wurde die QtcZeit engmaschig kontrolliert.

Ende 2008 beschrieb der Patient eine etwa $25 \%$ ige Verbesserung des Tremors; die Budipin-Dosis betrug zu dem Zeitpunkt 3-mal $20 \mathrm{mg}$ täglich. In der neurologischen Untersuchung zeigte sich neben einem intermittierend auftretenden Ruhetremor am rechten Arm auch ein rechtsbetonter mäßig ausgeprägter Rigor, eine geringe Verlangsamung der Fingermotilität beidseits, eine Reduktion der Mitbewegungen beidseits und eine geringe Hypomimie. Es wurde auch über einen vermehrten Speichelfluss berichtet.

2010 traten pektanginöse Beschwerden auf. Als Ursache fand sich eine koronare Herzerkrankung, weshalb im Juli 2010 eine Bypass-Operation durchgeführt werden musste. Schon einige Monate vorher war Budipin wegen seiner kardialen Nebenwirkungen und einem nicht zufriedenstellenden Effekt auf den Tremor abgesetzt und eine Therapie mit L-Dopa 3-mal 100/25 mg/Tag begonnen worden. Herr L. berichtete über eine leichte Verbesserung des Tremors, doch kam es im Anschluss an die Operation wieder zu einer relativ deutlichen Zunahme des Zitterns, das sich nun als grobschlägiger Ruhe- und Haltetremor manifestierte. Der Patient war dadurch speziell beim Essen, aber auch bei anderen Aktivitäten stärker beeinträchtigt, während sich die sonstige Parkinson-Symptomatik nicht verschlechtert hatte. Es wurde deshalb im November 2010 L-Dopa auf 3-mal 200/50 mg täglich gesteigert. Die Therapie mit Rasagilin war seit 2006 beibehalten worden. Unter dieser Einstellung kam es zu einer relativ deutlichen und für den Patienten zufriedenstellenden Verbesserung des Tremors, der nur mehr intermittierend vor allem bei Aufregungen auftrat.

Diese Verbesserung hält bis jetzt weiter an. Bis auf einen beidseitigen geringen Rigor, einen verminderten Armschwung rechts und eine leicht vorgebeugte Haltung ist keine sonstige Parkinson-Symptomatik bemerkbar.

Wegen einer etwas verkürzten Wirkungsdauer der einzelnen L-Dopa-Gaben wurde lediglich 2016 die Dosierung von 3-mal auf 4-mal täglich und auf eine
Tagesdosis von $700 \mathrm{mg}$ L-Dopa erhöht und damit die Dosierungsintervalle von 5 auf 4 Stunden reduziert. Auch unter dieser Dosis bestehen keine Nebenwirkungen. Der jetzt 80 -jährige Patient ist mit dieser Einstellung voll mobil und aktiv und in seinen vielen täglichen Aktivitäten in keiner Weise beeinträchtigt.

Letzte Parkinson-Therapie: Madopar ${ }^{\circledR}$ 200/50 1-1-1-1/2; Azilect ${ }^{\circledR}$ 1-mal morgens.

\section{Kommentar zu den Fallberichten}

Fall 1 illustriert in typischer Weise die in diesem Fall relativ rasch eintretenden Komplikationen der L-Dopa-Therapie bei einer Patientin mit frühem Krankheitsbeginn. Auffällig ist die positive Familienanamnese sowohl in der mütterlichen als auch väterlichen Linie, was auf eine wahrscheinliche genetische Ursache bzw. Prädisposition hinweist (evtl. rezessiver Erbgang, fragliche Parkinmutation). Eine primäre Behandlung mit dem Dopaminagonisten Pramipexol ret. war nur relativ kurzausreichend wirksam und führte in höherer Dosis zu einer Impulskontrollstörung mit nächtlicher Computer-Spielsucht, sodass bereits zwei Jahre nach Krankheitsbeginn mit einer L-Dopa-Therapie begonnen werden musste. Bei einer relativ hohen Dosis in Kombination mit dem COMT-Hemmer Entacapon traten einerseits starke Dyskinesien und andererseits psychiatrische Probleme mit Stimmungsschwankungen zwischen hypomanisch und depressiv auf, die mit den motorischen Schwankungen korreliert waren. Zuletzt konnte bei der jetzt 53-jährigen Patientin mit einer reduzierten L-Dopa-Dosis in Kombination mit dem COMT-Hemmer Tolcapon und einer niedrigen Dosis des atypischen Neuroleptikums Quetiapin eine einigermaßen zufriedenstellende Einstellung erreicht werden.

Fall 2 beschreibt einen Patienten mit einer benignen verlaufenden tremordominanten Parkinson-Erkrankung, die erst in höherem Alter aufgetreten war. Eine Behandlung mit Rasagilin und Pramipexol ret. war nicht ausreichend wirksam. Auch mit dem speziell gegen Tremor wirksamen Präparat Budipin psychopraxis.neuropraxis 2016 · 19:109-114 DOI 10.1007/s00739-016-0334-x

(c) Der/die Autor(en) 2016. Dieser Artikel ist eine Open-Access-Publikation.

\section{T. Brücke}

\section{Parkinson: L-Dopa nach wie vor wirksamste Therapie}

\section{Zusammenfassung}

Über 50 Jahre nach seiner Einführung in die klinische Praxis ist L-Dopa nach wie vor die wirksamste Therapie der ParkinsonErkrankung, an der sich alle anderen Parkinson-Medikamente messen müssen. Die beiden Fallberichte demonstrieren die gute Wirksamkeit und die Probleme einer mehrjährigen L-Dopa-Therapie. Die Ursachen dieser Probleme liegen in der Pharmakokinetik von L-Dopa in Kombination mit dem zunehmenden Verlust nigrostriärer dopaminerger Nervenzellen mit fortschreitender Erkrankung. Es besteht jedoch die Hoffnung, dass durch neue galenische Formen von L-Dopa diese Probleme reduziert werden können. Eines dieser Präparate steht knapp vor der Einführung, andere werden derzeit in klinischen Studien untersucht.

\section{Schlüsselwörter}

M. Parkinson - L-Dopa-Therapie · L-DopaLangzeitsyndrom . Pharmakokinetik . Fallberichte

\section{Parkinson's disease: L-dopa is still the most effective therapy}

\section{Abstract}

More than 50 years after its introduction into clinical practice, L-dopa still is the most effective therapy for Parkinson's disease and the gold standard for all other medications. In this article, two case reports are used to illustrate the good clinical efficacy and also the long-term complications of L-dopa therapy. The cause for these problems are the pharmacokinetics of L-dopa in combination with the progressive loss of nigrostriatal dopaminergic neurons in the course of the disease. New formulations of L-dopa offer hope for the improvement of these complications. One of these will soon be available for clinical use, while others are being tested in ongoing clinical trials.

\section{Keywords}

Parkinson's disease $\cdot \mathbf{L}$-dopa therapy $\cdot \mathbf{L}$-dopa long-term complications. Pharmacokinetics. Case reports 

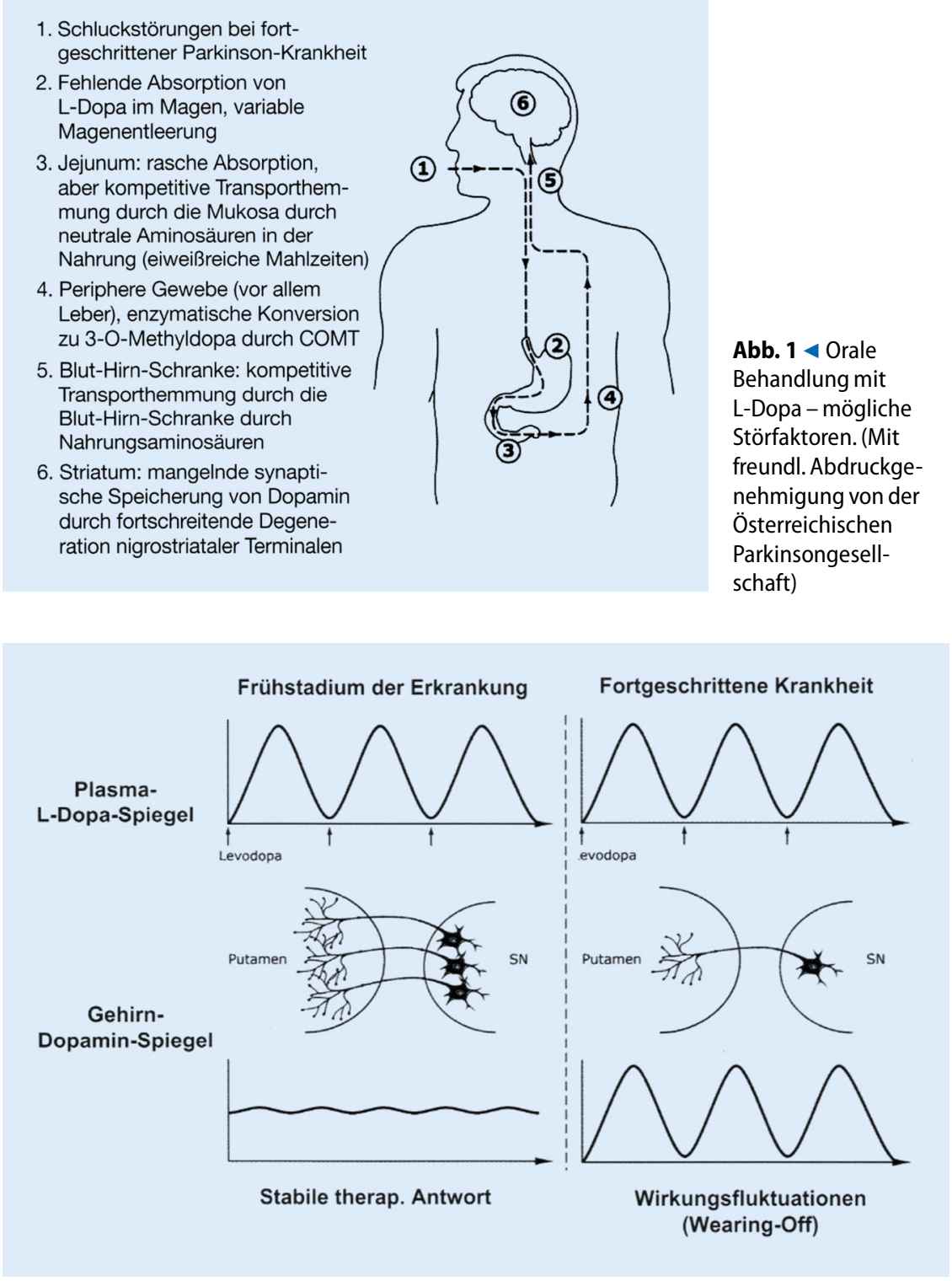

Abb. $2 \Delta$ Durch ungleichmäßige und pulsförmige Stimulation der Dopamin-Rezeptoren im Striatum werden Fluktuationen und Dyskinesien hervorgerufen (SN Substantia nigra). (Mit freundl. Abdruckgenehmigung von der Österreichischen Parkinsongesellschaft)

konnte nur eine passagere Verbesserung erreicht werden. Erst mit einer höheren L-Dopa-Dosis in Kombination mit Rasagilin wurde eine über Jahre zufriedenstellende Einstellung erreicht. Retrospektiv betrachtet wäre es sinnvoll gewesen, die Therapie schon primär mit L-Dopa zu beginnen. Dies ist wahrscheinlich wegen der kaum vorhandenen Bewegungsverlangsamung und der Tatsache, dass der Tremor häufig auf L-Dopa nicht gut anspricht, primär nicht versucht worden. Trotz der langjährigen L-Dopa-Therapie ist es zu keinen Langzeitkomplikatio- kurze Plasmahalbwertszeit von L-Dopa hervorgerufen.

Diese Schwankungen der L-DopaKonzentration im Plasma führen $\mathrm{zu}$ pulsatilen Schwankungen der L-DopaAufnahme ins Gehirn und damit zu Schwankungen der Dopamin-Freisetzung im Striatum, da aufgrund der zunehmenden dopaminergen Degeneration die Dopamin-Schwankungen dort nicht mehr abgepuffert werden können. Durch ungleichmäßige und pulsförmige Stimulation der Dopamin-Rezeptoren im Striatum werden Fluktuationen und Dyskinesien hervorgerufen (• Abb. 1 und 2).

Das Ziel der medikamentösen Parkinson-Therapie in diesem Stadium ist es daher, eine möglichst gleichmäßige und kontinuierliche Dopamin-RezeptorStimulation anzustreben.

\section{Magenentleerung und L-Dopa- Resorption, Fluktuation der Plasmaspiegel}

L-Dopa kann nicht im Magen, sondern erst im Duodenum und oberen Jejunum resorbiert werden, sodass der Magenentleerung eine entscheidende Rolle für die besondere Pharmakokinetik von L-Dopa zukommt. Die Magenentleerung wird durch eine Vielzahl von Faktoren beeinflusst und ist bei der ParkinsonErkrankung verlangsamt. Dies hängt mit degenerativen Veränderungen autonomer Nervenfasern in der Magenwand zusammen, die möglicherweise ganz am Beginn der neurodegenerativen Kaskade der Erkrankung stehen. Außerdem wird die Entleerung des Magens auch durch L-Dopa direkt verlangsamt.

Bei der Nahrungsaufnahme konkurriert L-Dopa mit aromatischen Aminosäuren beim Transport in das Blut und in das Gehirn. Eine proteinreiche Nahrung kann daher zu einer Verzögerung und Verminderung der L-Dopa-Resorption und Wirkung führen. Wenn möglich, sollte L-Dopa daher $1 / 2$ bis 1 Stunde vor bzw. 1 1 $1 \frac{2}{2}$ bis 2 Stunden nach den Mahlzeiten (insbesondere nach proteinreichen Mahlzeiten) eingenommen werden. Bei Patienten mit komplizierten Dosierungsschemata und häufiger Medikamenteneinnahme ist dies aber schwer durch- 
führbar und kann unter Umständen zu einer verminderten Nahrungs- und speziell Eiweißzufuhr führen. Das ist problematisch und sollte unbedingt vermieden werden, da Parkinson-Patienten im Verlauf der Erkrankung häufig an Gewicht verlieren und auch genügend $\mathrm{Ei}$ weiß zuführen sollten. Auch gibt es $\mathrm{Pa}$ tienten, bei denen nüchtern eingenommenes L-Dopa zu Übelkeit und Brechreiz führt, und Patienten, die keine Beeinflussung der mit den Mahlzeiten eingenommenen L-Dopa-Dosen bemerken. Die Empfehlung der Medikamenteneinnahme in fixen Abständen vor bzw. nach den Mahlzeiten muss daher im Einzelfall modifiziert werden. Die zusätzliche Gabe von Domperidon (Motilium $^{\circledR}$ ) kann das Auftreten von Übelkeit verhindern und auch die Magenentleerung beschleunigen. Auch fettreiche Ernährung kann zu einer Beeinflussung der L-Dopa-Resorption führen. Einige Studien berichteten über eine Beeinflussung der L-Dopa-Resorption bei Helicobacter-pyloriInfektion und eine damit verbundene Verschlechterung von motorischen Fluktuationen bzw. eine Verbesserung nach Eradikationstherapie. Das konnte allerdings in einer rezenten Untersuchung nicht bestätigt werden. Die gleichzeitige Einnahme von L-Dopa und Eisenpräparaten kann aufgrund einer Chelatbildung die Resorption von L-Dopa vermindern und sollte daher vermieden werden. Zu beachten ist auch, dass gleiche L-Dopa-Dosen bei Patienten mit niedrigem Körpergewicht oder höherem Alter zu höheren Plasmaspiegeln führen können.

\section{Zentrale Abpufferung der L-Dopa-Fluktuation}

Die Schwankungen der L-Dopa-Plasmaspiegel und die damit verbundenen Schwankungen der L-Dopa-Zufuhr im Striatum können im Frühstadium der Erkrankung bzw. bei nicht so ausgeprägtem Verlust dopaminerger Neurone ausgeglichen werden, da das aus L-Dopa gebildete Dopamin in noch intakten Nervenzellen gespeichert werden kann. Damit können die pulsatilen L-Dopa Spiegel abgepuffert werden. Beim Fortschreiten der Erkrankung und ausgeprägterer De- generation ist dies nicht mehr möglich und es kommt daher $\mathrm{zu}$ den typischen Komplikationen mit motorischen Fluktuationen, wobei die Wirkungsdauer von L-Dopa etwa der Halbwertszeit im Plasma entspricht (ca. 3 Stunden). Das Auftreten des L-Dopa-Langzeitsyndroms ist daher in erster Linie vom Ausmaß der dopaminergen Degeneration und nicht von der Dauer der L-DopaTherapie abhängig. (Im beschriebenen Fallbeispiel 1 ist anzunehmen, dass ein ausgeprägterer dopaminerger Zellverlust besteht als bei beim benigneren tremordominanten Fall 2).

\section{Therapie des L-Dopa- Langzeitsyndroms}

Das Ziel der medikamentösen Parkinson-Behandlung nach Auftreten von Komplikationen der L-Dopa-Therapie ist es daher, eine möglichst gleichmäßige und kontinuierliche Dopamin-RezeptorStimulation anzustreben. Eine Möglichkeit, dies zu erreichen, ist die Kombination von L-Dopa mit den länger wirksamen Dopaminagonisten. Die Retardpräparate (Ropinirol retard/Requip Modutab ${ }^{\circledR}$; Pramipexol retard/Sifrol ret. ${ }^{\circledR}$ ) mit einer Wirkung von bis zu 24 Stunden erscheinen in diesem Zusammenhang besonders günstig. Ein noch gleichmäBigerer und konstanterer Plasmaspiegel wird mit Rotigotin, einem Dopaminagonisten in Pflasterform erreicht.

$\mathrm{Da}$ das Risiko des Auftretens des L-Dopa-Langzeitsyndroms mit Fluktuationen motorischer und nicht motorischer Symptome und Dyskinesien bei jüngeren Patienten höher ist, gilt nach wie vor, dass diese Patienten (unter 65 Jahren bzw. biologisch junge Patienten) primär nicht mit L-Dopa, sondern je nach Schweregrad der Symptome z. B. mit MAO-B-Hemmern oder Dopaminagonisten behandelt werden sollten. Bei fast allen Patienten ist es aber früher oder später notwendig, L-Dopa zusätzlich zu geben, um eine gute Beweglichkeit zu erreichen (siehe Fall 1).

Es sollte diesbezüglich nicht zu lange zugewartet werden, da eine ausreichende Therapie der Bewegungsverlangsamung die Basis regelmäßiger körperlicher Aktivität bzw. sportlicher Betätigung ist und dadurch wahrscheinlich auch der Krankheitsverlauf positiv beeinflusst werden kann. Die früher bestehende Sorge, dass durch die L-Dopa-Therapie der Nervenzellverlust eventuell beschleunigt werden könnte, hat sich in keiner Untersuchung bestätigt. Bei älteren Patienten (über 70 Jahren, bzw. biologisch alten Patienten) besteht kein Grund mit der L-Dopa-Therapie zuzuwarten. Bei entsprechender Symptomatik sollte deshalb die Behandlung primär mit einem L-Dopa-Präparat beginnen. Bei dieser Patientengruppe ist auch zu beachten, dass Dopaminagonisten stärkere Nebenwirkungen, wie z.B. Blutdruckabfälle oder psychotische Symptome, auslösen können und man deshalb mit der Verabreichung dieser Substanzen vorsichtig sein muss!

Schwankende L-Dopa-Plasmaspiegel können bis zu einem gewissen Grad auch durch kürzere Dosierungsintervalle ausgeglichen werden. Die L-Dopa-Wirkung kann durch zusätzliche Gabe von COMT-Hemmern (Entacapon, Kombinationspräparat mit den Wirkstoffen L-Dopa, Carbidopa und Entacapon, Tolcapon), die den Abbau von L-Dopa bzw. Dopamin hemmen, etwas verlängert werden. Ebenso kann die Hemmung der Monoaminoxidase (MAO B) durch Verzögerung des Dopamin-Abbaus im Gehirn die L-Dopa-Wirkung verlängern und verstärken (Selegilin (Jumex ${ }^{\circledR}$ ), Rasagilin). Auch gibt es Hinweise für mögliche neuroprotektive Effekte der MAO-B-Hemmer. Die bisher verfügbaren L-Dopa-Retardpräparate haben leider die Hoffnungen nicht erfüllt, eine Verbesserung der motorischen Fluktuationen zu bewirken, und werden meist nur zur Therapie der nächtlichen Akinese eingesetzt.

\section{Neue L-Dopa-Präparate in der „Pipeline"}

In den letzten Jahren wurden zahlreiche neue galenische Formen von L-Dopa/ Carbidopa mit retardierter Wirkung untersucht. Eines dieser Präparate, bei dem die Wirksubstanzen aus Mikrosphären langsam freigesetzt werden, ist bereits in den USA im Handel (IPC066, Rytary ${ }^{\circledR}$ ) und auch schon in Europa zugelassen 
$\left(\right.$ Numient $\left.^{\circledR}\right)$. In den vorliegenden Studien konnte eine relativ deutlich verlängerte Plasmahalbwertszeit und damit verbunden eine Verlängerung der Wirkungsdauer und der Dosierungsintervalle und Abnahme der Häufigkeit der täglich nötigen Gaben (von 5 auf 3) sowie eine Zunahme der On-Zeiten von etwa einer Stunde gezeigt werden.

Weitere L-DOPA-Retardpräparate sind in klinischer Testung. Es gibt Berichte über eine subkutan kontinuierlich verabreichbare Form von L-Dopa/Carbidopa (ND0612) und ein inhalierbares L-Dopa/Carbidopa-Präparat in Pulverform, mit dem ein rascher Wirkungseintritt erreicht werden kann (CVT-301).

\section{Invasive Therapieverfahren}

Trotz dieser Maßnahmen können aber bei einem Teil der Patienten die Fluktuationen und/oder Dyskinesien mit oraler Therapie nicht zufriedenstellend behandelt werden. Diese Patienten schwanken zwischen Phasen völliger Unbeweglichkeit und Phasen mit massiven überschüssigen Bewegungen hin und her und sind unter Umständen nur wenige Stunden pro Tag einigermaßen gut beweglich. Für diese Patienten gibt es drei invasivere Therapieverfahren:

1. Die kontinuierliche Zufuhr von L-Dopa über eine Jejunal-Sonde mithilfe einer Pumpe (Duodopa ${ }^{\circledR}$ ).

2. Die kontinuierliche Verabreichung des Dopaminagonisten Apomorphin s. c. über Pumpe.

3. Die Tiefe Hirnsimulation mit stereotaktischer Implantation von Elektroden in den Nucleus subthalamicus bds. Mithilfe dieser Elektroden kann das falsche Entladungsmuster von Nervenzellen in diesem Kern durch Hochfrequenzstimulation korrigiert werden, wodurch sämtliche motorische Hauptsymptome der ParkinsonErkrankung deutlich verbessert werden können. Die tiefe Hirnstimulation kommt in erster Linie für Patienten vor dem 70. Lebensjahr infrage, die keine Hirnatrophie und keine Demenz aufweisen, während die Levodopa/Carbdopa-Pumpentherapie und unter Umständen auch die Apomorphin-Pumpentherapie auch bei älteren und dementen Patienten infrage kommt.

\section{Nicht motorische Symptome}

Im Verlauf der Parkinson-Erkrankung tritt neben den motorischen Symptomen auch eine große Zahl nicht motorischer Symptome auf, wie z. B. Demenz, Depression, Angst und Panikattacken, Schmerz und vegetative Symptome wie orthostatische Dysregulation, Blasenfunktionsstörung und Schweißausbrüche. Diese Beschwerden beeinträchtigen die Lebensqualität der Patienten oft stärker als die motorische Behinderung. Als Nebenwirkung der dopaminergen Therapie können Halluzinationen oder Impulskontrollstörungen (z. B. Spiel-, Kauf- oder Sexsucht) auftreten. Alle diese nicht motorischen Symptome sind erst in den letzten Jahren in den Mittelpunkt des Interesses gerückt und stellen eine besondere therapeutische Herausforderung dar.

\section{Fazit für die Praxis}

- L-Dopa ist die wirksamste Therapie der Parkinson-Erkrankung.

- Es gibt keine Hinweise auf eine Krankheitsbeschleunigung bzw. Neurotoxizität durch L-Dopa.

- L-Dopa ist das Mittel der 1. Wahl bei Parkinson-Patienten $>70$ Jahre.

- Bei Parkinson-Patienten < 65 Jahre sollte die Therapie primär mit MAOB-Hemmern bzw. Dopaminagonisten - in Abhängigkeit vom Schweregrad der Symptome - beginnen. Bei unzureichender Wirkung sollte aber zusätzlich so rasch als nötig die Gabe von L-Dopa erfolgen.

- Bei motorischen Fluktuationen und/ oder Dyskinesien ist eine möglichst kontinuierliche dopaminerge Stimulation durch häufigere L-DopaGaben, die Kombination mit Dopaminagonisten, MAO-B-Hemmern bzw. COMT-Hemmern anzustreben.

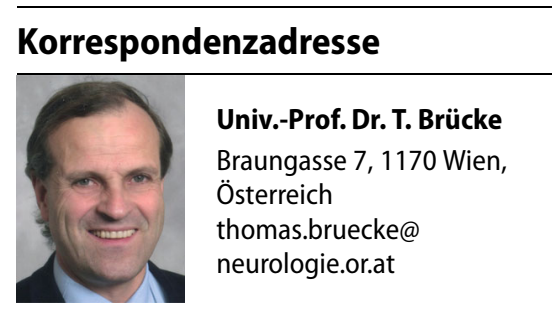

Open access funding provided by Medical University of Vienna.

\section{Einhaltung ethischer Richtlinien}

Interessenkonflikt. T. Brücke gibt an, dass kein Interessenkonflikt besteht.

Dieser Beitrag beinhaltet keine von den Autoren durchgeführten Studien an Menschen oder Tieren Die Fallberichte ermöglichen keine Identifikation der beschriebenen Patienten, weshalb keine schriftliche Einwilligung eingeholt wurde. Die Anfangsbuchstaben der Patienten sind fiktiv.

Open Access Dieser Artikel wird unter der Creative Commons Namensnennung 4.0 International Lizenz (http://creativecommons.org/licenses/by/4.0/deed. de) veröffentlicht, welche die Nutzung, Vervielfältigung, Bearbeitung, Verbreitung und Wiedergabe in jeglichem Medium und Format erlaubt, sofern Sie den/die ursprünglichen Autor(en) und die Quelle ordnungsgemäßnennen, einen Linkzur Creative Commons Lizenz beifügen und angeben, ob Änderungen vorgenommen wurden. 